\title{
Faits et fiction dans le reportage littéraire : à propos du Shah de Ryszard Kapuściński
}

Facts and Fiction in Literary Reportage: about Shah of Shahs by Ryszard Kapuściński

\section{Beata Nowacka}

Traducteur : Agnieszka Grudzińska

\section{(2) OpenEdition}

Journals

Édition électronique

URL : https://journals.openedition.org/recherchestravaux/3504

DOI : $10.4000 /$ recherchestravaux.3504

ISSN : 1969-6434

Éditeur

UGA Éditions/Université Grenoble Alpes

Édition imprimée

ISBN : 978-2-37747-301-4

ISSN : 0151-1874

Référence électronique

Beata Nowacka, « Faits et fiction dans le reportage littéraire : à propos du Shah de

Ryszard Kapuściński », Recherches \& Travaux [En ligne], 98 | 2021, mis en ligne le 28 juin 2021,

consulté le 09 août 2021. URL : http://journals.openedition.org/recherchestravaux/3504 ; DOI :

https://doi.org/10.4000/recherchestravaux.3504

Ce document a été généré automatiquement le 9 août 2021.

(c) Recherches \& Travaux 


\title{
Faits et fiction dans le reportage littéraire : à propos du Shah de Ryszard Kapuściński
}

\author{
Facts and Fiction in Literary Reportage: about Shah of Shahs by \\ Ryszard Kapuściński
}

\section{Beata Nowacka}

Traduction : Agnieszka Grudzińska

1 Ryszard Kapuściński a-t-il, grâce à son immense talent, réinventé le reportage ? Est-il plutôt un auteur de fiction? La question de la véracité des faits qu'il relate dans ses reportages suscite, depuis plusieurs années, de vifs débats parmi les lecteurs et les journalistes. On pourrait espérer que les experts des sujets abordés par le reporter polonais viennent ici éclairer un public quelque peu désorienté. Seulement, il existe autant d'opinions que d'experts ${ }^{1}$. Voici les avis de deux éminents spécialistes de l'Iran sur Le Shah, reportage que Ryszard Kapuściński a consacré à la révolution iranienne ${ }^{2}$ :

\footnotetext{
Abbas Milani :

Ouvrez Le Shah à n'importe quelle page, et je vous dirai ce qui est faux ou partiellement faux ${ }^{3}$.

Hooman Majd :

Il n'y a rien d'étonnant à ce que seuls les Polonais, parmi les habitants des pays occidentaux, comprennent si bien l'Iran et son peuple, vu qu'un excellent livre sur la révolution islamique de 1979 - un livre bien meilleur que tous les textes commis par les auteurs non seulement occidentaux mais aussi iraniens - a été écrit par un Polonais dont les œuvres ne cessent de m'inspirer. Je pense évidemment à Ryszard Kapuściński ${ }^{4}$.
}

2 Ces deux opinions sur Le Shah ont été exprimées à peu près au même moment par deux Iraniens appartenant à une même génération, tous deux issus de familles riches, éduqués en Europe et aux États-Unis. Aujourd'hui, tous deux sont des spécialistes reconnus de l'Iran. Majd a travaillé entre autres pour The New York Times, The New Yorker et Newsweek. Milani, quant à lui, est professeur d'université. Tous deux sont également auteurs de publications importantes consacrées à leur pays. Leur vie a été 
fortement marquée par les événements rapportés dans Le Shah. Hooman Majd est en effet le petit fils de l'ayatollah Mohammad Kazem Assar et le fils d'un diplomate ayant travaillé pour le dernier shah. Abbas Milani, quant à lui, a donné dans les années 1975-1977 des cours à l'Université de Téhéran. Arrêté par la SAVAK (police secrète) pour ses opinions de gauche, il a été incarcéré dans la même prison que les futurs chefs de la République islamique d'Iran. Majd et Milani habitent et travaillent aux États-Unis.

3 Les opinions divergentes de ces deux experts du domaine iranien révèlent des conceptions différentes de la vérité. Toutes les deux doivent cependant être abordées avec la même prudence. Ce n'est pas un hasard si l'affirmation de Majd est tout aussi catégorique que dénuée de fondement. Il faut savoir qu'elle est tirée de l'introduction à l'édition polonaise de son livre, Demokracja ajatollahów. Wyzwanie dla Iranu [La démocratie des ayatollahs. Un défi pour l'Iran] : elle relève d'une courtoisie proprement iranienne. Il en va autrement pour Milani : interrogé sur la valeur factuelle du Shah par Artur Domosławski, auteur d'une célèbre - et controversée - biographie de Kapuściński, Milani indique quelques imprécisions plus ou moins importantes, tout en soulignant que les erreurs sont nombreuses, puisqu'on peut en trouver à chaque page du texte. Domosławski accepte cette affirmation sans la vérifier et s'en sert pour mettre en cause la véracité non seulement du Shah, mais également de tous les autres reportages de Kapuściński.

4 Je n'ai pas l'intention d'entamer ici une polémique avec le biographe. J'aimerais simplement montrer que sa méthode, qui consiste à se référer à une autorité, est trompeuse. En effet, on peut toujours trouver un autre expert qui n'est pas du même avis. Voir clair dans un brouillamini d'inexactitudes plus ou moins importantes, voulues ou accidentelles, peut s'avérer chronophage et complexe. Cela demande des recherches poussées qui, pour être fiables, nécessitent souvent la connaissance de plusieurs langues. Pourtant, il convient d'examiner, plus attentivement que ne le fait Domosławski, les critiques adressées à Kapuściński par les spécialistes de l'Iran. Voici un exemple pour s'en convaincre : Milani estime inexacte la phrase suivante : « Le shah était décidé à garder son trône et, pour ce faire, il explora toutes les possibilités ${ }^{5} . . . »$ Selon le chercheur, le shah n'était pas si déterminé. Pourtant, le shah a plusieurs fois exprimé sa résolution sur ce sujet. Cinq ans avant la révolution, par exemple, il avait confié à Oriana Fallaci :

Il arrive fréquemment que le roi en ait assez d'être roi. Cela ne signifie pas que moi, je veux y renoncer, je crois trop en la personne que je suis et en ce que je fais. [...] La monarchie est le seul moyen possible pour gouverner l'Iran. [...] Je considère [...] avoir une mission que je dois remplir jusqu'au bout, et j'ai bien l'intention de le faire, sans renoncer au trône ${ }^{6}$.

Il faut prendre avec la même précaution les remarques de Milani, citées par Domosławski, concernant le fait que Kapuściński aurait été «manipulé »: «[...] il avait des amis à gauche et au centre gauche qui lui ont raconté leur version des faits ${ }^{7}$ ». Milani affirme par exemple que Kapuściński «[à] un moment, évoque des milliers de prisonniers tués par le shah, or sous son régime, ce sont quelque mille quatre cents prisonniers qui ont été exécutés ${ }^{8} »$. En réalité, cette information - «des milliers de prisonniers assassinés »- ne figure pas dans Le Shah. De même, Milani, lui-même arrêté au moment des faits, se souvient que Kapuściński parle de « centaines de milliers de prisonniers politiques ${ }^{9} "$, alors que ces derniers n'étaient que 4500 . En réalité, le reporter a simplement écrit : «Il y avait dans les prisons, selon l'opposition, cent mille 
prisonniers politiques ${ }^{10}$.» Même si on ne prend pas en compte la prudente formule "selon l'opposition", utilisée par le reporter, on voit bien que Milani et Domosławski gonflent considérablement les chiffres avancés dans Le Shah. Vérifions. Dans l'ouvrage de référence, L'Histoire de l'Iran, on lit: « [...] si les données officielles iraniennes pour les années 1976-1977 estimaient le nombre de prisonniers politiques à environ trois mille, selon les estimations d'Amnesty International, ils étaient en réalité entre vingt-cinq et cent mille ${ }^{11} »$. Enfin, la dernière critique de Milani concerne l'ignorance $\mathrm{du}$ reporter quant aux finances de l'Iran, et notamment ses remarques sur «les centaines de millions de dollars sur lesquels les courtisans ont fait main basse ${ }^{12} »$. Un autre spécialiste éminent de l'Iran, Ervand Abrahamian, évalue la fortune du shah à un milliard de dollars, et celle de la famille royale à plus de vingt milliards ${ }^{13}$. Milani conclut que Kapuściński extrapole la fortune du shah en se fondant sur quelques phrases sorties de leur contexte, voire sur des affirmations en réalité absentes du texte. Elles n'apportent pas grand-chose au débat sur la technique du reporter, ni à celui sur la littérature du réel.

6 Cela ne signifie pas pour autant que Le Shah est un livre dépourvu d'inexactitudes et d'erreurs. Les exemples que j'ai choisi d'analyser concernent des détails sortis de leur contexte. Ils peuvent cependant nous aider à repérer chez l'auteur du Shah une certaine logique dans l'organisation des faits. C'est cette logique que j'aimerais mettre ici en évidence. Tout d'abord je vais analyser les inexactitudes les plus faciles à repérer et sans importance pour le message global. Dans un deuxième temps, je vais commenter quelques erreurs factuelles involontaires, pour considérer ensuite des exemples de brouillage délibéré du contour des événements et de l'espace, procédé que Kapuściński utilise pour donner un sens symbolique et littéraire aux faits. J'analyserai ensuite des exemples qui témoignent de la fidélité du reportage de Kapuściński à des textes, images et voix éphémères et apparemment de moindre importance mais émanant bien de l'univers iranien. J'en viendrai pour finir à l'analyse de la dernière partie du Shah, écrite deux années plus tard, où apparaissent des parallèles entre ce qui s'est passé en Iran et en Pologne (et ailleurs), qui confèrent une dimension essayistique au reportage et débordent la question de la vérité factuelle.

7 Les amateurs d'erreurs factuelles et autres inexactitudes trouveront dans Le Shah plusieurs exemples savoureux témoignant d'une rédaction peu soignée et d'une vérification insuffisante de la part du reporter. Pour prendre un exemple, lorsqu'il évoque la décision de Reza Chah de changer le nom Perse pour Iran, Kapuściński mentionne une information souvent citée mais fausse que l'on peut trouver dans plusieurs ouvrages, y compris scientifiques: «En 1935 - changement du nom historique Perse pour $\operatorname{Iran}^{14} »$. En réalité, il ne s'agit pas exactement d'un changement de nom soudain. En 1935, Reza Chah a adressé une note officielle à la Société des Nations et aux États pour demander l'abandon du nom Perse pour celui d'Iran (c'est-à-dire le pays des Aryens), endonyme utilisé par les habitants du pays depuis l'antiquité et qui, étymologiquement, dérive d'un adjectif indo-iranien signifiant «noble ${ }^{15}$ ». La décision du shah a sans doute été motivée par le nationalisme grandissant dans le pays, provoqué à son tour par un relâchement de la dépendance vis-à-vis de la GrandeBretagne (la dynastie des Pahlavi, récemment fondée, y a beaucoup contribué). En 
imposant cette appellation, le shah souhaitait promouvoir l'idée d'un État moderne et rompre avec l'image peu reluisante de pays arriéré qui s'était imposée au XIXe siècle sous la domination des Kadjars. Les tendances pro-allemandes qui s'intensifiaient alors en Iran ont également encouragé ce changement d'appellation : il s'agissait de mettre en avant le caractère soi-disant « aryen » du pays, au sens d'indo-européen.

Une autre inexactitude peut être reprochée à Kapuściński, lorsqu'il agrège trois biographies en une seule, en prétendant brosser le portrait du colonel Liakhov :

Il est coiffé d'une toque en astrakan noir, car, comme je viens de le dire, il est officier de la Brigade cosaque persane (l'unique armée que possède alors le shah), commandée par le colonel tsariste de Saint-Pétersbourg, Vladimir Liakhov qui aime les soldats de métiers. Or notre jeune officier en est le type même. [...] Mais les grandes promotions ne commencent à tomber qu'après 1917, quand le shah, qui suspecte Liakhov de sympathies bolcheviques (à tort, du reste), le licencie et le renvoie en Russie. Reza Khan est alors promu colonel et commandant de la Brigade cosaque qui se trouve être protégée par des Anglais ${ }^{16}$.

Vladimir Platonovitch Liakhov a bien été colonel de l'armée russe, commandant de la brigade cosaque persane (la garde de la famille royale) et gouverneur militaire de la capitale dans les années 1908-1909. C'est sous son commandement que la division cosaque (ex-brigade) a, en juin 1908, pris d'assaut et occupé Téhéran de juin 1908 à juillet 1909. Cependant, contrairement à ce qu'avance Kapuściński, Liakhov n'a pas été accusé par le shah de sympathies pro-bolchéviques, ni limogé et renvoyé en Russie. En 1917, année mentionnée par Kapuściński, le commandement de la brigade cosaque était assuré par le colonel Gueorgui Iossifovitch Klerje (Clergé) un Français au service du tsar, effectivement soupçonné de penchants bolchéviques ${ }^{17}$. Le shah l'a bien limogé et remplacé, cependant non par le père du dernier shah d'Iran (comme l'écrit Kapuściński), mais par le colonel Vsevolod Dmitrievitch Starosselski. Ce dernier a certes permis au jeune Reza Chah de monter rapidement en grade, mais c'est seulement en 1920, une fois Starosselski écarté, que Reza Chah a pris la tête de la division.

Une autre inexactitude concerne les armes commandées par le shah en 1978. Selon Kapuściński :

Un jour, en feuilletant un catalogue d'usine d'armement, le monarque tombe en extase devant destroyer Spruance. Le prix d'un seul exemplaire s'élève à 338 millions de dollars. Le shah en commande aussitôt quatre. Les chasseurs arrivent par bateau à Bandar Abbas [...]. Aujourd'hui encore, ces chasseurs se dégradent dans le port de Bandar Abbas ${ }^{18}$.

11 En réalité, les destroyers ne se dégradent pas dans le port Bender Abbas, pour la simple raison qu'ils n'y sont jamais parvenus. Leur production a commencé aux États-Unis en 1970 et leur prix a initialement été fixé entre 60 et 115 millions de dollars. En 1974, l'Iran a envisagé d'en acheter six unités mais a finalement renoncé à l'achat de deux d'entre elles. Les quatre destroyers restants, commandés le 23 mars 1978, n'ont jamais été remis au shah. Après la victoire de la révolution islamique, lorsque l'acheteur potentiel était en passe de devenir un ennemi, la transaction a été suspendue. Deux jours après le retour de Khomeiny à Téhéran, le nouveau pouvoir a renoncé à l'achat de deux destroyers et, le 31 mars, il a abandonné l'idée d'acheter les deux suivants ${ }^{19}$.

Un autre doute encore surgit dans le dernier chapitre du Shah. Le reporter y décrit le chaos qu'il découvre dans une rue de Téhéran alors qu'il se dirige vers l'ambassade des États-Unis, un soir de décembre 1979 : lambeaux de banderoles et fragments d'affiches 
de propagande jonchent le sol. Sur l'un d'eux, le reporter lit l'inscription : «Ali, l'imam inspiré, se préparait au martyr ${ }^{20} »$. Or, Ali ibn Abi Talib (601-661), cousin et gendre de Mahomet (Muhammad ibn Abd Allah) a été blessé en 661 par un fanatique de la secte des kharijites à l'entrée d'une mosquée iraquienne et en est mort quelques jours plus tard, le 25 janvier $661^{21}$. Il est permis de supposer que le reporter a vu sur cette image révolutionnaire non pas Ali ibn Abi Talib, mais son fils, Hussein (vers 626-680) qui, lui, a bien dû se préparer au martyr, conscient qu'il ne survivrait pas au combat contre l'armée, supérieure en nombre, des Omeyyades. Il est tombé sur le champ de bataille de Karbala le 10 octobre 680 . Cette date est centrale pour la martyrologie shiite et les commémorations du martyr d'Hussein - les ta'zieh - ont joué un rôle clé dans l'esthétique sombre de la révolution islamique. Les opposants au régime du shah se sont en effet approprié le ta'zieh: leurs manifestations, semblables à des processions funèbres, cherchaient à imiter ces célèbres représentations religieuses ${ }^{22}$.

Les inexactitudes mentionnées ci-dessus discréditent-elles Le Shah en tant que chronique de la révolution de 1978-1979 ? Certes, les imprécisions suscitent des doutes. Elles sont sûrement, dans une certaine mesure, délibérées : elles vont de pair avec le désordre de la chambre d'hôtel où le reportage sur le chaos révolutionnaire a vu le jour. Il est cependant indéniable qu'un certain nombre d'erreurs factuelles - le changement du nom du pays, les détails sur les armes acquises par le shah, ou les informations biographiques - auraient pu être évitées. Je considère néanmoins que leur présence dans le texte n'entame pas la valeur objective de ce reportage.

La méthode du biographe de Kapuściński, Artur Domosławski, s'avère inadéquate dans le cas d'erreurs que Kapuściński a délibérément introduites dans le texte, en effaçant à dessein les contours factographiques ${ }^{23}$. On peut donner comme exemple la scène initiale du Shah, lorsque le reporter commente des photographies, qu'il a pu voir mais qui ne sont reproduites dans aucune des éditions de l'ouvrage, afin de décrire les visages des principaux antagonistes. On y trouve des mots en majuscules qui se démarquent du restant du texte comme les titres sur la page d'un journal : «IL EST PARTI $^{24}$ !», «IL EST REVENU ${ }^{25}$ !». Non seulement l'auteur ne précise pas qui est représenté sur ces photographies, mais il déroute à dessein le lecteur. Il est pourtant certain qu'il disposait des éditions spéciales des journaux iraniens les plus populaires, tels que Kayhan ou Ettelâ'ât, dont les unes annonçaient: Szah raft (le shah est parti) ou Emam amad ('Imam est arrivéé). Le reporter ne dit mot du contexte dans lequel ont été prises ces photographies. Elles documentent pourtant des événements qui ont attiré l'attention du monde entier et qui en ont changé la face pour quelques décennies: la fuite du shah Mohammad Reza Pahlavi qui le 16 janvier 1979 a définitivement quitté le pays, ainsi que le retour en Iran, deux semaines plus tard à peine, de l'ayatollah Khomeiny après un exil de plusieurs années. Pourquoi Kapuściński épure-t-il ainsi son propos? Pourquoi cherche-t-il à désorienter le lecteur et à rendre difficile son cheminement au milieu des faits? Peut-être parce qu'il veut que le lecteur, tout comme le reporter dans sa chambre d'hôtel en Iran, éprouve de l'incertitude face la mosaïque iranienne, enchevêtrée et fragmentaire.

Kapuściński ne se comporte pas non plus en journaliste professionnel lorsqu'il puise sans scrupules dans les rumeurs et les ragots. C'est le cas, par exemple, lorsqu'il décrit le prétendu ancêtre du futur shah d'Iran en se fiant à une mention anonyme légendant une photographie :

C'est la photo la plus ancienne que j'ai réussi à me procurer. Elle représente un soldat qui tient, de la main droite, une chaîne à laquelle est attaché un homme. Le 
soldat et l'homme enchaîné regardent l'objectif avec concentration, on voit que c'est un moment important pour eux. Le soldat est un homme âgé, de petite taille, le type même du paysan simple et soumis ; il est mal fagoté, son uniforme est trop grand pour lui, son pantalon tombe en accordéon sur ses pieds, son calot de guingois est retenu par ses oreilles en feuilles de chou, bref, il a l'air comique, il fait penser au brave soldat Chvéik. L'homme enchaîné (visage émacié et blême, yeux enfoncés) a la tête bandée, il est manifestement blessé. Une légende sous la photo précise que le soldat est l'aïeul du shah Mohammad Reza Pahlavi (le dernier souverain d'Iran) et que l'homme blessé est le meurtrier du shah Nassereddin. Le cliché remonte à 1896, quand, à l'issue de quarante-neuf années de règne, Nassereddin fut assassiné par l'homme que l'on voit sur la photo ${ }^{27}$.

Dans cette description de photographie, la première de celles qui composent le chapitre intitulé «Daguerréotypes ", il n'est pas dit que Nassereddine Chah (1848-1896) ait été assassiné par Mirza Reza Kermani ${ }^{28}$ (1847-1896). On nous dit en revanche que le soldat qui escortait l'assassin était le grand-père de Mohammad Reza Pahlavi. Pourtant, cela est impossible. Abbas Ali-chah a certes été soldat (il a combattu lors de la guerre contre l'Afghanistan en 1856) et il a bien fondé la dynastie des Pahlavi, mais il est subitement mort en novembre 1878, presque 20 ans avant l'assassinat de Nassereddine. Et même s'il avait vécu jusqu'en 1896, il aurait eu plus de 80 ans au moment de l'assassinat, plusieurs dizaines d'années de plus que le soldat de la photographie (semblable au brave soldat Chvéik).

La description de la rue Ferdousi, qui clôt le tableau de l'Iran, constitue un autre exemple d'effacement des contours de la réalité :

Quand je veux me remonter le moral et passer un moment agréable, je me rends rue Ferdousi où Monsieur Ferdousi tient une boutique de tapis persans. Monsieur Ferdousi, qui a passé sa vie entière à côtoyer l'art et la beauté, regarde la réalité qui l'entoure comme un film de série $\mathrm{B}$ dans un cinéma minable et sale. «Mon cher Monsieur, tout est une affaire de goût, c'est ce qui importe le plus. Le monde aurait une autre allure si les gens étaient un peu plus nombreux à avoir du goût, me dit-il, il faut avoir du goût, c'est ce qui importe le plus. Toutes les horreurs (car pour lui ce sont de véritables horreurs), le mensonge, la trahison, le vol, la délation, ont un dénominateur commun : elles sont commises par des gens dénués de goût. » Il croit que le peuple survivra et que la beauté est indestructible. « Il faut que vous vous souveniez, me dit-il en déroulant un tapis (il sait pertinemment que je ne l'achèterai pas mais il me le montre pour le plaisir des yeux), que ce qui a permis aux Perses de rester des Perses pendant deux mille cinq cents ans, ce qui nous a permis de rester nous-mêmes, en dépit de toutes les guerres, les invasions, les occupations, c'est notre force spirituelle, et non pas notre force matérielle, notre poésie, et non pas notre technique, notre religion, et non pas nos usines. Qu'avons-nous donné au monde ? La poésie, les miniatures et les tapis. Comme vous le voyez, les choses les plus inutiles du point de vue de la production. Mais c'est à travers elles que nous nous sommes exprimés. Nous avons donné au monde des merveilles superflues, uniques, irremplaçables[... $]^{29} »$.

L'auteur décrit sa conversation avec le mystérieux marchand de tapis en évitant de donner la moindre information d'ordre topographique. Le lecteur aura tendance à imaginer que la petite boutique de monsieur Ferdousi est nichée dans une petite ruelle que les habitants ont baptisée du nom du marchand. Or, il existe bien une rue Ferdousi (ou Ferdowsi) à Téhéran. C'est une grande artère commerciale, véritable axe financier du pays. On y trouve le siège de la Banque Nationale d'Iran (Bank Melli Iran) ainsi que de nombreux bureaux de change, officiels et officieux. Ce n'est certainement pas un 
lieu où M. Ferdousi se serait senti à l'aise, lui qui appréciait tant « la puissance du bon goût ${ }^{30}$ ».

Pourquoi Kapuściński dissimule-t-il des faits importants, pourquoi brouille-il leurs contours, pourquoi cherche-t-il à désorienter le lecteur en ne lui donnant que des bribes d'informations équivoques? Grâce à ce procédé, le lecteur peut éprouver dans sa propre chair les émotions du reporter qui peine à décrire le chaos révolutionnaire. L'évocation de l'Iran au moyen de descriptions de quelques photographies (que le lecteur ne peut pas voir) constitue un procédé littéraire. Même si ces descriptions ne correspondent pas à la vérité historique, elles disent quelque chose du souverain en place. La photographie représentant le soldat et l'homme enchaîné donne lieu au récit de l'épuisant voyage que ceux-ci ont accompli pour se rendre dans la capitale où l'homme doit être exécuté. Ce récit suggère l'idée d'un effort solennel, qu'on peut comprendre comme l'expression d'une détermination inébranlable. L'ekphrasis présente ainsi deux avantages. Premièrement, l'histoire de la dynastie Pahlavi, période cruciale de l'histoire de l'Iran moderne, n'est pas racontée sous la forme d'un cours monotone mais se raconte en quelque sorte toute seule, au moyen d'anecdotes où interviennent des protagonistes incarnés et vivants. Deuxièmement, l'auteur interroge les dessous de la grande histoire en permettant à son lecteur d'apercevoir les fragments qui la composent tout en la laissant insaisissable : les émotions humaines, le rôle des sens, les mentalités. On peut interpréter de la même manière les procédés qui visent à dissimuler la réalité triviale de la rue Ferdousi. Si Kapuściński a choisi de donner ce nom à son marchand de tapis, c'est assurément en souvenir d'Abul-Qâsem Ferdowsi, poète persan, qui a donné son nom à la rue ${ }^{31}$. Il crée ainsi un Homère persan, qui devient une métaphore de l'Iran, de son histoire glorieuse, de son apport au patrimoine culturel mondial et de ses valeurs inaliénables. Pour Kapuściński, ces valeurs-là, et non la réalité révolutionnaire, peuvent devenir le lieu d'une rencontre durable et fructueuse.

\section{II}

Toutes ces analyses pourraient conduire à la conclusion que Le Shah est, comme l'a suggéré Abbas Milani, rempli d'erreurs élémentaires facilement repérables à chaque page du livre. En réalité, malgré toutes ses imprécisions, Le Shah contient un remarquable savoir sur l'Iran, attesté par d'autres sources. Je vais maintenant aborder la question de la fiabilité du reportage, en analysant quelques documents sur la genèse de l'ouvrage.

Dans sa description de la révolte islamique, Kapuściński a surtout mis en avant le caractère iconique de celle-ci. La description évocatrice de la chambre en désordre du reporter, où s'entasse la documentation pour le reportage à venir, rend parfaitement compte du caractère impréparé, chaotique et parfois véritablement mystique de la révolution de 1978-1979, qui s'est nourrie - elle a probablement été la première à le faire avec une telle intensité - de la force des médias. La révolte n'est-elle pas partie des appels de Khomeiny enregistrés sur des cassettes magnétophoniques distribuées sous le manteau pour être écoutées pendant les meetings et dans les foyers? Les esprits ne se sont-ils pas échauffés grâce aux images transmises en continu par les télévisions et aux photographies omniprésentes exhibant les victimes du régime, leurs bourreaux et les portés disparus? 
22 J'ai pu, sans trop de difficultés, retrouver les photographies qui sont décrites par Kapuściński. Certaines étaient accessibles sur Internet; d'autres dans des publications en langue anglaise consacrées à l'histoire de l'Iran moderne ${ }^{32}$. De même, il a été relativement aisé d'évaluer la véracité de certains éléments contextuels convoqués par l'auteur. Par exemple, lorsque Kapuściński mentionne l'entretien au cours duquel le shah informe les journalistes sur le sort des prisonniers politiques, il constate: «En septembre 1978, quatre mois avant son départ, le shah accorde une interview à un correspondant de l'hebdomadaire Stern ${ }^{33}$. " L'entretien avec le shah a en effet été publié dans le numéro 36 de l'hebdomadaire allemand et le texte cité correspond mot pour mot à celui qui a été publié dans la presse ${ }^{34}$. Kapuściński cite aussi littéralement une autre interview du shah d'Iran, publiée quelques années plus tôt, le 7 janvier 1974, dans l'hebdomadaire Der Spiege ${ }^{35}$. Sur la couverture de ce numéro, on pouvait lire le titre: Ölkriese: Gespräch mit dem Schah ${ }^{36}$ [Crise pétrolière : conversation avec le shah].

L'auteur tient également à ce que les événements que vivent les protagonistes de son livre trouvent leur ancrage dans la réalité. Ainsi, on force un certain Mahmud, un Iranien qui revient d'exil, à devenir membre du parti du shah, le Rastakhiz ${ }^{37}$. Comme il résiste, on lui donne un tract où figurent les lignes suivantes, attribuées au monarque : «CEUX QUI N'ADHÈRENT PAS AU RASTAKHIZ SONT SOIT DES TRAÎTRES DONT LA PLACE EST EN PRISON SOIT DES ÉLÉMENTS QUI NE CROIENT NI AU SHAH NI AU PEUPLE NI À LA PATRIE ET NE PEUVENT, PAR CONSÉQUENT, ESPÉRER ÊTRE TRAîTÉS À L'ÉGAL DES AUTRES $^{38}$ ». En créant cette scène, qui montre le sort d'un Iranien ordinaire, le reporter se sert d'un savoir qu'il puise dans des documents de cette époque. Le shah a tenu des propos similaires à plusieurs reprises et Kapuściński a pu tomber, par exemple, sur son discours publié dans Iran Yearbook 1977/2353 : «Ceux qui n'adhéreront pas à ces structures politiques et qui ne croient pas aux trois piliers dont j'ai parlé, ont deux possibilités. Si une personne appartient à une organisation politique clandestine, dite "de masse" dans notre langue [tude en persan, cf. le nom du parti communiste iranien] elle est alors dite apatride. La place de cette personne est dans une prison iranienne. Ou alors, si elle le souhaite elle peut, dès demain, recevoir un passeport et partir [...], puisqu'elle n'est pas de nationalité iranienne. Elle séjourne en Iran de manière illégale, ce que la loi sanctionne par des peines prévues dans ces cas-là ${ }^{39}$. "

Un procédé semblable est à l'œuvre lorsque, pour rendre les vibrations de la rue iranienne, le reporter convoque l'image des manifestants entonnant un chant plein d'expression et de pathos - Allah Akbar -, dans lequel un refrain dit :

Iran, Iran, Iran

Chun-o-marg-o-osjan.

(Iran, Iran, Iran,

Mort, révolte et sang ${ }^{40}$ )

25 Les paroles de ce chant ont été écrites par Afshin Sarfaraz et la musique, composée par Feridun Chochnud. Le chant est devenu très populaire dans les années 1978-1979, dans une interprétation de Reza Rujgarie. Il a le rythme d'une marche. On trouve aujourd'hui, sur Internet, plusieurs films sur la révolution iranienne qui le reprennent comme musique de fond. Kapuściński cite fidèlement l'extrait.

Ces exemples visent avant tout à souligner le caractère visuel et sonore de la révolution islamique telle que la décrit Kapuściński. En faisant un tel choix, l'écrivain a décidé d'exposer au premier plan la dimension éphémère du coup d'État iranien. Mais ne faudrait-il pas ajouter ici : de chaque coup d'État? Dans cette chronique, nous n'avons 
pas affaire à un savoir tiré d'un manuel mais aux voix de la rue livrée à la révolution, aux affects et aux émotions qui accompagnent les tournants politiques: la peur qui monte et qui s'éteint, la joie de la dignité retrouvée, ou bien les sentiments d'épuisement et d'impuissance, qui ont couronné plus d'une insurrection.

Le dernier chapitre du reportage, intitulé "La flamme morte ", a été écrit après un intervalle de deuxans pendant lequel Kapuściński a pris part aux événements d'août 1980 en Pologne. La valeur factuelle de ce chapitre pourrait éveiller beaucoup de doutes chez le lecteur à la recherche d'une description fiable des événements de l'époque. Contrairement à ses reportages antérieurs, Kapuściński ne se soucie plus de donner une représentation claire de ce moment important de l'histoire de l'Iran. Riche des expériences de la "révolution polonaise de $1980-1982^{41}$ ", pour reprendre la formule de Timothy Garton Ash, il s'efforce de saisir le phénomène de la révolution en tant que telle. Il y voit une bataille entre deux forces : la structure et le mouvement. Il précise :

Le mouvement attaque la structure, aspire à sa destruction, la structure quant à elle se défend, veut détruire le mouvement. Aussi puissantes l'une que l'autre, ces deux forces ont des caractéristiques différentes. Ce qui caractérise le mouvement c'est sa spontanéité, son impétuosité, son dynamisme et sa fugacité. La structure en revanche, se distingue par sa force d'inertie, sa résistance, sa faculté étonnante, instinctive presque, de survie ${ }^{42}$.

L'action conjuguée de ces deux forces propres à la révolution est repérable dans toute la structure du Shah. Le mouvement, c'est donc à la fois le chaos du bureau du reporter, l'invasion des images dans les médias, le vacarme de la rue de Téhéran et celui des nouvelles diffusées par la radio. On peut également situer dans cet espace instable les silhouettes des leaders et les événements courants que Kapuściński a transmis dans ses dépêches envoyées à l'Agence Polonaise de Presse (PAP) ${ }^{43}$. Le rôle de la structure serait en revanche joué par des éléments pérennes et intemporels, cachés sous la surface des événements : les images de la condition humaine, les émotions, les modèles de pouvoir, et, avant tout, les valeurs durables qui donnent accès à l'altérité. Dans Le Shah, ce rôle est joué par la culture iranienne. Elle est personnifiée par monsieur Ferdousi admirant la beauté des tapis persans. Cet homme plein de noblesse, créé sans doute sur le modèle de M. Cogito, héros d'un cycle poétique du poète Zbigniew Herbert ${ }^{44}$, fait commerce de tapis, et ce n'est pas un hasard: dans la langue persane, le mot «tapis » ne signifie-t-il pas « collection des poésies ${ }^{45} »$ ?

\section{III}

Le Shah a été publié dans l'hebdomadaire varsovien Kultura trois ans avant la publication en volume. Le premier épisode est paru le 29 juillet 1979. Le travail sur le livre a été interrompu par un nouveau départ du reporter en Iran afin de couvrir pour l'Agence Polonaise de presse (PAP) l'occupation de l'ambassade américaine par les partisans de Khomeiny Kapuściński a repris le travail sur le livre seulement deux années plus tard, juste avant l'instauration de l'état de siège, le 13 décembre 1981. Les dernières livraisons ont commencé à paraître, dans le même hebdomadaire, en août 1981.

Il est difficile d'imaginer un reportage sans erreurs qui décrirait les circonstances de la révolution islamique à chaud, sous la forme d'un feuilleton hebdomadaire, par un 
auteur non-spécialiste de l'Iran, ne disposant pas d'Internet. Il faut avoir à l'esprit que la révolution islamique a surpris le monde entier : l'idéologie islamo-marxiste, fondée par Ali Shari'ati (1933-1977) a non seulement joué un rôle important dans la formation de la société révolutionnaire, mais s'est également avérée attractive pour la population urbaine misérable et désorientée comme pour l'intelligentsia à la recherche d'idées nouvelles. Guy Sorman, auteur d'un essai intitulé Comment finissent les révolutions ${ }^{46}$ rappelle le soutien que la gauche internationale a apporté au mouvement de Khomeiny, notamment des intellectuels français tels Jean-Paul Sartre qui a manifesté contre le shah, ou Michel Foucault qui voyait dans la figure d'ayatollah « le libérateur du peuple iranien ${ }^{47}$ ».

31 Kapuściński, s'est attaqué à un sujet difficile même pour les esprits les plus avisés, et cela sans préparation aucune. Les témoignages de ceux qui ont pu le côtoyer pendant qu'il travaillait sur son livre n'en sont que plus précieux. Nous disposons d'un témoignage intéressant de Marek Smurzyński, co-auteur de l'excellent volume Historia Iranu [Histoire de l'Iran], cité ci-dessus, et traducteur de poésie polonaise et persane, professeur de l'Université Jagellon de Cracovie et de celle de Téhéran. Lorsque Kapuściński travaillait sur Le Shah, Smurzyński était étudiant et militait activement dans l'opposition contre le régime communiste. Son témoignage sur Kapuściński a été diffusé par la radio Zamaneh, émettant d'Amsterdam, le 28 janvier 2007. À la question posée par Pejman Akbarzade concernant les connaissances de Kapuściński sur la culture de l'Iran et la langue persane, Marek Smurzyński répond :

L'histoire de la découverte de l'Iran par Kapuściński est très intéressante. Quand il est parti en Iran, j'étais encore étudiant. [...] À son retour, nous l'avons invité à l'université pour l'entendre parler de son expérience iranienne. Nous ne connaissions pas du tout ce pays et étions curieux de savoir à quoi il ressemblait. [...] C'était la première rencontre de Kapuściński avec l'Iran. Quand il travaillait sur Le Shah, il essayait d'être très attentif, rencontrait volontiers des Iraniens qui habitaient en Pologne, les écoutait pour tirer le meilleur de ce qu'ils lui racontaient ${ }^{48}$.

La genèse du livre de Kapuściński a eu lieu à une époque où le savoir sur l'Iran n'était pas facilement accessible puisque même dans le milieu universitaire le témoignage du reporter était appréhendé comme une source fiable. Il ne faut pas s'attendre à ce que ce livre soit exempt d'erreurs. Certaines inexactitudes y sont même volontaires. La force de ce témoignage ne réside pas dans sa valeur factographique, mais bien dans l'originalité de sa perspective. Le Shah transmet au lecteur contemporain non seulement un savoir important sur l'histoire ancienne et moderne de l'Iran, mais également les désarrois du journaliste s'efforçant de décrire l'histoire en cours, la vie d'un homme pris dans ses mécanismes et, enfin, la force de la littérature qui, dans une réalité étrangère, permet de retrouver le modèle universel d'un événement historique. 


\section{NOTES}

1. Je remercie Mateusz M. Kłagisz de l’Université Jagellon de Cracovie pour son aide concernant le domaine iranien. Une version légèrement modifiée de cet article a été publiée en polonais dans la revue Świat $i$ Słowo [Le monde et la parole], $\mathrm{n}^{\circ} 34,2020$. Disponible en ligne: <https:// swiatislowo.publisherspanel.com/resources/html/article/details?id=206567> [consulté le 25/11/2020].

2. Dans cet article j'utilise les éditions suivantes : en polonais, R. Kapuściński, Cesarz, Szachinszach [1982], Varsovie, Czytelnik, 1990; en français: Le Shah, traduit du polonais par V.Patte dans R. Kapuściński, Euvres, Paris, Flammarion, 2010. Je donne d'abord la référence dans la traduction française puis, entre parenthèses, dans la version originale.

3. Cité par A. Domosławski, dans Kapuściński, préfacé par J. Krauze, traduit du polonais par L. Dyèvre, Paris, Les Arènes, 2011, p. 391. En polonais : Kapuścinski non-fiction, Varsovie, Świat KsiĄżki, 2010, p. 421.

4. H. Majd, Demokracja ajatollahów. Wyzwanie dla Iranu [La démocratie des ayatollahs. Un défi pour l'Iran], traduit de l'anglais par D. Żukowski, Cracovie, Karakter, 2011, p. 11.

5. «Szach był zdecydowany, żeby utrzymać siĘ na tronie, i aby to osiAgnAzć, próbował wszystkich możliwości... », Domosławski, ouvr. cit., p. 42. La phrase est identique dans le livre de Kapuściński : voir p. 275 dans la version polonaise (en français : p. 391).

6. O. Fallaci, Wywiad z historiA [Entretien avec l'histoire], traduit de l'anglais par A. Czepnik et coll., Varsovie, Weltbild Polska, 2012, p. 24-31. (L'ouvrage d'O. Fallaci a également été traduit en français : Entretiens avec l'histoire, Paris, Flammarion, 1975).

7. A. Domosławski, ouvr. cit., p. 392 (en polonais : p. 422).

8. Ibid.

9. Ibid.

10. Le Shah, ouvr. cit., p. 89 (p. 201).

11. A. Krasnowolska (dir.), Historia Iranu [Histoire de l'Iran], Wrocław, Ossolineum, 2011, p. 868.

12. A. Domoslawski, ouvr. cit., p. 392 (p. 422).

13. E. Abrahamian, Historia wspótczesnego Iranu, [Histoire de l'Iran moderne], traduit de l'américain par N. Nowak, Varsovie, Książka i Wiedza, 2011, p. 174.

14. E. J. Osmańczyk, Encyklopedia Spraw MiĘdzynarodowych i ONZ, [Encyclopédie des affaires internationales et de l'ONU], Varsovie, PWN, 1974, p. 351.

15. A. Krasnowolska, ouvr. cit., p. 827.

16. Le Shah, ouvr. cit., p. 37-38 ("Na głowie ma kozackA papachĘ, z czarnych karakułów, gdyż, jak wspomniałem, jest oficerem Brygady Perskich Kozaków (jedynej armii, jakA posiada wówczas szach), którA dowodzi carski pułkownik z St. Petersburga Wsiewołod Liachow. Reza Khan jest pupilem pułkownika Liachowa, który lubi urodzonych żołnierzy, a nasz młody oficer jest typem urodzonego żotnierza [...]. Wielkie awanse zaczynajĄ jednak sypać siĘ dopiero po roku 1917, kiedy to szach posĄdzajĄc Liachowa (zupełnie niesłusznie) o sympatie dla bolszewików, zwalnia go ze służby i odprawia do Rosji. Teraz Reza Khan zostaje pułkownikiem i dowódcą kozackiej brygady, którĄ od tej chwili opiekują się Anglicy. », p. 168).

17. A. Krasnowolska, ouvr. cit., p. 811.

18. Le Shah, ouvr. cit., p.115-116 ("Oto któregoś dnia monarcha oglądając prospekty firm zbrojeniowych wpadł $w$ zachwyt na widok najnowszego niszczyciela Spruance. Cena jednego egzemplarza wynosiła trzysta trzydzieści osiem milionów dolarów. Szach natychmiast zamówił cztery. Niszczyciele przypłynĘły do portu Bender Abbas [...]. Owe niszczyciele niszczejA do dziś w porcie Bender Abbas.", p. 218). 
19. Voir J. Krzewiński, « Amerykańskie niszczyciele typu SPRUANCE et KIDD » [« Les torpilleurs américains du type SPRUANCE et KIDD»], Nowa Technika Wojskowa, [La Nouvelle Technique Militaire], $\mathrm{n}^{\circ}$ 5, 1997, p. 46-53 et 1997, n 6, p. 54-60.

20. Le Shah, ouvr. cit., p. 207 (« natchniony imam Ali przygotowywał siĘ do mĘczeńskiej śmierci », p. 278).

21. A. Krasnowolska, ouvr. cit., p. 336.

22. Ibid., p. 878-879, p. 881.

23. Je traite plus amplement cette question dans un article intitulé «"Każda rewolucja to zmaganie siĘ dwóch sił: struktury i ruchu". Gramatyka rewolucji w Szachinszachu Ryszarda Kapuścińskiego » [ "Toute révolution est une lutte entre deux forces : la structure et le mouvement". Grammaire de la révolution dans Le Shah de Ryszard Kapuściński »], Białostockie Studia Literaturoznawcze, $\mathrm{n}^{\circ} 11,2017$, p. 231-241.

24. Le Shah, ouvr. cit., p. 13 (« ODLECIAE », p. 153).

25. Ibid. (« WRÓCIE »).

26. Voir E. et A. Lisowski, Południk szczĘścia [Le méridien de la chance], Cracovie, 2016, p. 20-21.; A. Milani, The Shah, New York, St Martin's Press, 2011, p. 414.

27. Le Shah, ouvr. cit., p. 33 ("Jest to najstarsze zdjęcie, jakie udało mi siĘ zdobyć. Widać na nim żołnierza, który w prawej ręce trzyma łańcuch, do łańcucha przywiĄzany jest człowiek. Żołnierz i człowiek na łańcuchu patrzA $w$ obiektyw ze skupieniem, widać, że jest to dla nich ważna chwila. Żołnierz jest starszym mĘżczyznĄ niskiego wzrostu, jest typem prostego i posłusznego chłopa, ma na sobie przyduży, niezgrabnie uszyty mundur, spodnie marszczĄ mu siĘ w harmonijkĘ, wielka, krzywo założona czapa opiera siĘ na odstających uszach, w ogóle wygląda zabawnie, przypomina Szwejka. Człowiek na łańcuchu (twarz szczupła, blada, oczy zapadniĘte) ma głowĘ owiniĘtĄ bandażem, widocznie jest ranny. Podpis pod zdjĘciem mówi, że ten żołnierz jest dziadkiem szacha Mohammeda Rezy Pahlaviego (ostatniego władcy Iranu), a ten ranny jest zabójcĄ szacha Naser-ed-Dina. Fotografia musi wiĘc pochodzić z roku 1896, kiedy to Naser-edDin, po czterdziestu dziewiĘciu latach panowania, został zabity przez widocznego na zdjĘciu mordercĘ. ", p. 165).

28. Voir C. Ghani, Iran and the Rise of Reza Shah, Londres/New York, 1998, p. 161-162; M. X. Schwerda, «Death on Display. Mirza Riza Kirmani, Prison Portraiture and the Depiction of Public Executions in Qajar Iran ", Middle East Journal of Culture and Communication, vol. VIII, $\mathrm{n}^{\circ} 2-3$, 2015, s. 172-191, [en ligne:] <https://www.academia.edu/19707582/ Death_on_Display._Mirza_Riza_Kirmani_Prison_Portraiture_and_the_Depiction_of_Public_Executions_in_Qajar_Iran> [consulté le 20/03/2019].

29. Le Shah, ouvr. cité, p. 239-240 (« Kiedy chcĘ poprawić sobie nastrój i spĘdzić przyjemnie czas, idĘ na ulicĘ Ferdousi, przy której pan Ferdousi prowadzi sprzedaż perskich dywanów. Pan Ferdousi, który całe życie spĘdził obcujĄc ze sztukĄ i piĘknem, patrzy na otaczajĄcą go rzeczywistość jak na drugorzĘdny film w tanim i zaśmieconym kinie. Wszystko jest kwestiĄ smaku, mówi mi, najważniejsza rzecz, proszĘ pana trzeba mieć smak. Świat wyglądałby inaczej, gdyby trochĘ wiĘcej ludzi miało odrobinĘ wiĘcej smaku. Wszystkie okropności (bo nazywa to okropnościami), takie jak kłamstwo, zdrada, złodziejstwo, donosicielstwo, sprowadza do wspólnego mianownika - takie rzeczy robią ludzie, którzy nie majĄ smaku. Wierzy $w$ to, że naród przetrwa wszystko $i$ że piĘkno jest niezniszczalne. Musi pan pamiĘtać, mówi mi rozwijając kolejny dywan (którego wie, że nie kupiE, ale chciałby, abym nacieszył nim oko), że to, co Persom pozwoliło pozostać Persami przez dwa i pót tysiąca lat, to, co pozwoliło nam pozostać sobAc, mimo tylu wojen, inwazji i okupacji, to była nasza siła duchowa, a nie materialna, nasza poezja, a nie technika, nasza religia, a nie fabryki. Co myśmy dali światu? Myśmy dali poezjĘ, miniaturĘ $i$ dywan. Jak pan widzi, z wytwórczego punktu widzenia same bezużyteczne rzeczy. Ale właśnie w tym wyraziliśmy siebie. Myśmy dali światu tĘ cudownĄ, niepowtarzalnĄ bezużyteczność. », p. 299-230).

30. Kapuściński se réfère ici sans doute au célèbre poème de Zbigniew Herbert, «La puissance du goût » (« PotĘga smaku »), paru dans la revue clandestine Zapis en 1981 ( ${ }^{\circ}$ 18, p. 5) quelques mois 
avant la publication des dernières parties du Shah dans l'hebdomadaire Kultura. En français : «La puissance du goût ", Zbigniew Herbert, Monsieur Cogito. CEuvres poétiques complètes II, traduit du polonais par Brigitte Gautier, Paris, Le Bruit du Temps, 2012, p. 437.

31. Abul-Qâsem Ferdowsi (932/941-1020/1025) est l'auteur de l'épopée nationale iranienne intitulée Shahnameh [Le Livre des rois] qui compte près de 60000 distiques et raconte dans la convention mythico-héroïque l'histoire de l'Iran, depuis la création du monde jusqu'à l'invasion arabe. L'épopée est élaborée sur la base d'écrits, de textes officiels, d'informations transmises oralement et d'histoires populaires. Cf. A. Krasnowolska, «Epos perski I jego polski przekład» [«L'épopée perse et sa traduction polonaise »], dans Livre des rois. Shahnameh, vol. I, traduit du persan par W. Dulદ̨ba, Cracovie, NOMOS, 2004, p.XXI. Voir aussi : M. Składankowa, «Mity królewskiej księgi. Symbole i wzorce mityczne w „Szahname” ", [ Les mythes du livre royal. Symboles et modèles mythiques dans Shahnameh»], Varsovie, Wydawnictwo Uniwersytetu Warszawskiego, 1981, p. 16-17 et L'Histoire de l'Iran, ouvr. cit., p. 485.

32. Cf. par ex.: < http://trunt.blogspot.com/2013/12/mirza-reza-kermani-c1850-1896.html> [consulté le 20/03/2018].

33. Le Shah, ouvr. cit., p. 97 («We wrześniu 1978, na cztery miesiĄce przed swoim odejściem, szach udziela wywiadu korespondentowi tygodnika», Stern, p. 206).

34. F. Tartarotti, R. Winter, «Das ganze Land kämpft. Stern-interview mit dem Schah von Persien » [« Le pays tout entier combat. Entretien avec le shah de Perse »], Stern, $n^{\circ}$ 36, 1978.

35. Le Shah, ouvr. cit., p. 99 (p. 210).

36. W. Knips, J. K. Engel, D. Wild „Ich werde Ihnen Aspirin verkaufen" [ "Je vais vous vendre de l'aspirine »], Der Spiegel, $\mathrm{n}^{\circ} 1 / 2,1974$, p. 24-31.

37. Rastakhiz: le Parti de la renaissance du peuple iranien. Après la liquidation du parti d'opposition, Parti Paysan, en 1975, le shah a fondé le parti monarchiste dont l'objectif principal était de promouvoir les réformes de la Révolution Blanche. Il est devenu le seul parti légal en Iran et en être membre était en principe obligatoire (les récalcitrants étaient menacés de prison ou condamnés à l'exil). Le parti a été dissout par le shah à la fin de 1978. Cf. N. R. Keddie, Wspótczesny Iran. Źródła i konsekwencje rewolucji [L'Iran contemporain. Les sources et les conséquences de la révolution], p. 156-157 ; A. Krasnowolska, ouvr. cit., p. 856, 858, 881.

38. Le Shah, ouvr. cit., p. 156 ( Ci, którzy nie bĘdĄ należeć do partii Rastakhiz, sĄ albo zdrajcami, dla których miejsce jest $w$ wiĘzieniu, albo nie wierzĄ $w$ Szacha, Naród i OjczyznĘ i dlatego nie powinni oczekiwać, że bĘdĄ traktowani na równi z innymi. », p. 245).

39. Iran Yearbook 1977/2353, Téhéran, 1977, p. 68. Voir aussi Rafi' Ettela'at-e 80 sal, vol. I, Téhéran, 1353, p. 296. Je dois cette information ainsi que la traduction du persan à Mateusz M. Kłagisz de l'Université Jagellon de Cracovie.

40. Le Shah, ouvr. cité., p. 97 (« petnA ekspresji i patosu pieśń - Allah Akbar, w której kilka razy powtarza siĘ refren: Iran, Iran, Iran/ Chun-o-marg-o-osjan./ (Iran, Iran, Iran,/ to krew, śmierć i bunt) », p. 206.

41. T. G. Ash, The Polish Revolution: Solidarity. 1980-82, New York, Scribner, 1984.

42. Le Shah, ouvr. cit., p. 217 (« Ruch atakuje strukturĘ, dĄży do jej zniszczenia, struktura broni się, chce unicestwić ruch. Obie te siły, jednako potĘżne, mają różne właściwości. WłaściwościĄ ruchu jest jego spontaniczność, żywiołowa, dynamiczna ekspansywność i - krótkotrwałość. Natomiast właściwościA struktury jest bezwładność, odporność, zdumiewająca, niemal instynktowna zdolność przetrwania.», p. 285).

43. R. Kapuściński a envoyé des dépêches à la PAP entre mars 1979 et mi-avril, et ensuite entre décembre 1980 et février 1980.

44. En français : Z. Herbert, Monsieur Cogito, ouvr. cité.

45. Comme le précise Władysław Dulęba, auteur d'une anthologie en trois volumes de poésie persane, Dywan perski, [Le tapis persan, p. 8], Cracovie, 1977, p. 8: «Le tapis est un recueil de poèmes d'un seul poète, ces poèmes sont agencés par genre et par ordre alphabétique, selon la 
dernière lettre du monorime ». Voir aussi J.W. von Goethe, Divan occidental-oriental [West-östlicher Divan], 1819.

46. Voir G. Sorman, Les Enfants de Rifaa. Musulmans et modernes, Paris, Fayard, 2003. Voir également M. Abassy, Kultura wobec postĘpu i modernizacji: Rosja i Iran w perspektywie porównawczej [La culture face au progrès et à la modernisation: la Russie et l'Iran dans la perspective comparatiste], Cracovie, éditions de l'Université Jagellon, 2013, p. 505-525.

47. Voir G. Sorman, ouvr. cit. Dans un article intitulé «Le retour du Prophète?», daté du 22/10/1978, Michel Foucault, correspondant du Corriere della Sera à Téhéran, met face à face l'image de Khomeiny, vu comme "un dirigent mythique de la révolution » et le personnage du shah. Il les voit tous les deux comme les héros des anciennes icônes ornées des blasons : «[...] le roi et le saint, le monarque armé et l'exilé sans défense, un despote et un homme adoré par les foules et qui lève ses mains au ciel. C'est une image très suggestive et elle répond à la réalité attestée par la mort de millions de personnes ». M. Foucault, Taccuino persiano/Cahier persan, R. Guolo, P. Panza (éd.), Guerini e Associati, Milan 2002, p. 10, 36, 59. L'engagement de Foucault dans la révolution iranienne est analysé par Didier Eribon dans Michel Foucault (1926-1984), Paris, Flammarion, coll. «Champs/Biographie », 1989. Voir aussi Michel Foucault, "Les reportages d'idées », Corriere, 1978 et Dits et écrits, III, p. 706-707 : «À quoi rêvent les Iraniens? " dans Le Nouvel Observateur, 16-22 octobre 1978, qui condense les articles de la première série parue dans le Corriere et reprend l'article "Retour au prophète", Corriere, 22/10/1978, (Dits et écrits, III, 688-694).

48. Voir < http://zamaaneh.com/special/2007/01/post_122.html> [consulté le 11/03/2019]. Je remercie Mateusz M. Kłagisz de m'avoir informée de l'existence de cette émission et d'avoir traduit du persan l'extrait cité.

\section{RÉSUMÉS}

Le Shah de Ryszard Kapuściński est un livre controversé. Les avis des spécialistes de l'Iran divergent à son sujet. Cet article sélectionne quelques descriptions d'événements de l'histoire iranienne et les met en parallèle avec des documents, notamment relatifs à la révolution de 1978-79. C'est ainsi que l'analyse des extraits du livre, comparés avec les événements historiques, permet de faire ressortir des inexactitudes factographiques, des erreurs évidentes, mais aussi des écarts voulus que l'auteur a sciemment insérés dans son texte. Les qualités du reportage de Kapuściński ne sont donc pas l'objectivité et la factualité mais l'universalité.

Shah of Shahs by Ryszard Kapuściński is considered as a controversial book-the opinions expressed on it by Iranian experts are definitely divergent. The author attempts to compare presentation of selected facts from the history of Iran, which are described in the report, to the information gathered in other (also academic) publications on the topic. Referring to the analysis of the passages of the book, which were compiled with relevant historical information, there were identified various types of factual inaccuracies-ranging from obvious factual errors to discrepancies implemented by the reporter deliberately. The greatest value of Kapuściński's report is though not the objectivity of the message but its universality. 
INDEX

Keywords : Shah of Shahs, Kapuściński, history, Iran, literary report

Mots-clés : Le Shah, Kapuściński, histoire, Iran, reportage littéraire

\section{AUTEURS}

\section{BEATA NOWACKA}

Université de Silésie

Beata Nowacka, professeur à l'Université de Silésie, est l'auteur de Ryszard Kapuściński. Biographie d'un écrivain [en collaboration avec Z. ZiĄtek, aux éditions Peter Lang, 2008]. Le livre a également été traduit en espagnol et en italien. Elle a également publié Literatura non-fiction. Czytanie Kapuścińskiego po Domosławskim [La littérature non-fiction. Lire Kapuściński après Domosławski] aux éditions de l'Université de Silésie. Elle a établi une édition critique du Shah de R. Kapuściński dans la collection «Biblioteka Narodowa » [Bibliothèque Nationale], ainsi que des nouvelles de Melchior Wańkowicz. 